\title{
Experimental demonstration of the induction-shimming concept in superconductive undulators
}

\author{
D. Wollmann, ${ }^{*}$ A. Bernhard, P. Peiffer, and T. Baumbach \\ University of Karlsruhe, Kaiserstrasse 12, 76131 Karlsruhe, Germany \\ E. Mashkina \\ University of Erlangen-Nürnberg, Staudtstrasse 3, 91058 Erlangen, Germany \\ A. Grau and R. Rossmanith \\ Forschungszentrum Karlsruhe, P.O. Box 3640, 76021 Karlsruhe, Germany
}

(Received 7 November 2008; published 23 April 2009)

\begin{abstract}
In an undulator, magnetic field errors limit the quality of the emitted photon beam. In an ideal undulator, the sinusoidal motion of the electron beam and the emitted photons are in phase along the whole trajectory. Small variations in the magnetic field from period to period can produce phase errors and have to be corrected. This procedure is called shimming. Recently, a new scheme for superconductive undulators based on Faraday's law of induction in a type-II superconductor was proposed in which these errors are automatically minimized. The field errors create currents and therefore magnetic fields in an array of coupled high temperature superconductor loops which minimize the field errors. In this paper the first experimental results of a test of this new concept are described.
\end{abstract}

DOI: 10.1103/PhysRevSTAB.12.040702

\section{INTRODUCTION}

Undulators are the photon sources in synchrotron light sources and free electron lasers [1,2]. The wavelength of the emitted light is related to the undulator period length $\lambda_{u}$ by the equation

$$
\lambda_{n}=\frac{\lambda_{u}}{2 n \gamma^{2}}\left(1+\frac{K^{2}}{2}\right)
$$

where $K=0.0934 \cdot \lambda_{u}[\mathrm{~mm}] \cdot \tilde{B}[\mathrm{~T}], n=1,3,5, \ldots$ is the harmonic number, and $\tilde{B}$ is the peak magnetic field. Deviations in $\tilde{B}$ and $\lambda_{u}$ due to mechanical tolerances can influence the trajectory of the particles and thereby disturb the constructive interference of the photons. Therefore mechanical tolerances limit the achievable brightness of the undulator radiation. This is especially true for higher harmonics. The measure for the field variations is the socalled phase error [1]. In permanent magnet undulators, the phase error is minimized by mechanical or magnetic shimming systems [1,2]. In superconductive undulators additional superconductive correction coils can be used for phase error correction [3-6].

Recently, a novel concept called induction shimming was proposed, in which the field errors for superconductive undulators are automatically minimized by an array of high temperature superconductor (HTSC) loops $[7,8]$. In this paper the first experimental results are reported.

\footnotetext{
*daniel.wollmann@cern.ch
}

PACS numbers: 41.75.Ht, 41.20.Gz, 74.78.Bz, 84.71.Ba

\section{INDUCTION SHIMMING}

The idea of induction shimming is sketched in Fig. 1. The induction-shimming system consists of a set of overlapping closed loops each covering two adjacent magnet poles. The array of HTSC loops is attached to the surface of the undulator. Each loop covers one full period and is electrically isolated from all other loops. For an ideal undulator field, the flux through one loop is zero. In case of deviations each half-wave has a slightly different field integral. For the sake of simplicity, the sinusoidal magnetic field in each half period of the undulator is approximated by a rectangular shape. Each rectangle $u_{1}, u_{2}, \ldots$ represents the integrated field of a half period. A more detailed calculation of this effect with sinusoidal fields can be found in [7]. In the case that the two undulator half periods covered by one loop have slightly different field amplitudes, a current is induced in the loop when the undulator

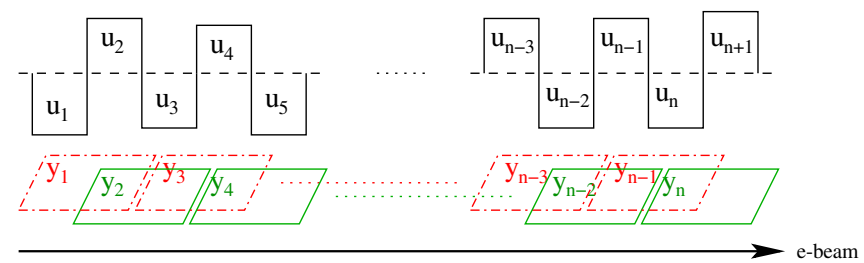

FIG. 1. (Color) Concept of induction shimming. The sinusoidal field in one of the undulator halves is approximated for the sake of simplicity by a rectangular shape. Because of minor field errors caused by finite tolerances, the amplitude of the field varies slightly. This variation is compensated by HTSC loops arranged in the way shown in this picture. The length of one loop in the beam direction is exactly one ideal period length. 
current is switched on. The magnitude of the induced current is described by Faraday's law:

$$
\oint_{C} \overrightarrow{\mathbf{E}} d \vec{l}=-\frac{d}{d t} \int_{S} \overrightarrow{\mathbf{B}} d \vec{A}
$$

where $\overrightarrow{\mathbf{B}}$ is the magnetic flux density over the area $S$ with the contour $C, \overrightarrow{\mathbf{E}}$ is the electrical field strength, and $d \vec{A}$ is the surface element. If the conductor along the contour $C$ is a superconductive closed-loop, Eq. (2) is reduced to

$$
0=\frac{d}{d t} \int_{S} \overrightarrow{\mathbf{B}} d \vec{A}
$$

If the externally produced magnetic flux through the loop changes by the amount of $\Delta \Phi$ a current is induced. The current produces a magnetic flux $-\Delta \Phi$ which compensates the change of the external magnetic flux. Because of the coupling, the field amplitude in each period is leveled to the same value. This idea is the base of the inductionshimming concept for undulators [7].

\section{EXPERIMENT}

In order to perform a first experimental test of this concept an existing superconductive undulator mock-up was used. The period of the undulator mock-up is $14 \mathrm{~mm}$. Each groove is filled with 28 round $\mathrm{NbTi}$ wires with a diameter of $0.8 \mathrm{~mm}$. The cross section of the wire is $0.5 \mathrm{~mm}^{2}$. From former measurements it was known that this mock-up has a rather deformed field (Fig. 2) mainly due to massive magnetic iron plates at the beginning and the end of the mock-up. This deformed field is well suited to study this effect. In order to reduce the field error seven $\mathrm{YBa}_{2} \mathrm{Cu}_{3} \mathrm{O}_{7-x}$ (YBCO) loops (see Fig. 3) were placed at

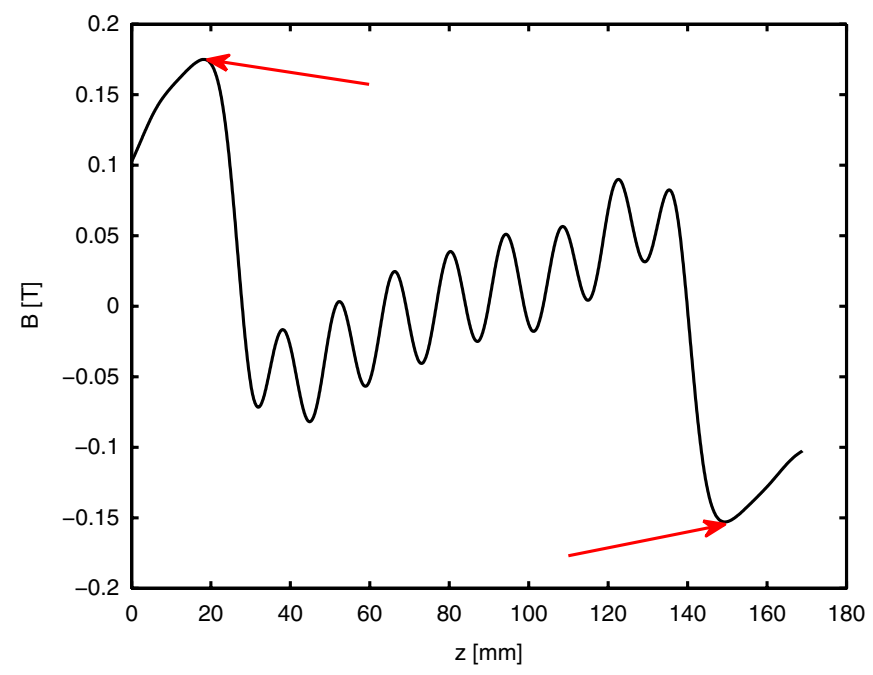

FIG. 2. (Color) The distorted magnetic field strength of an undulator mock-up before applying the induction-shimming concept. The field is significantly deformed by the end plates marked by the arrows. The period length of the undulator mockup is $14 \mathrm{~mm}$.

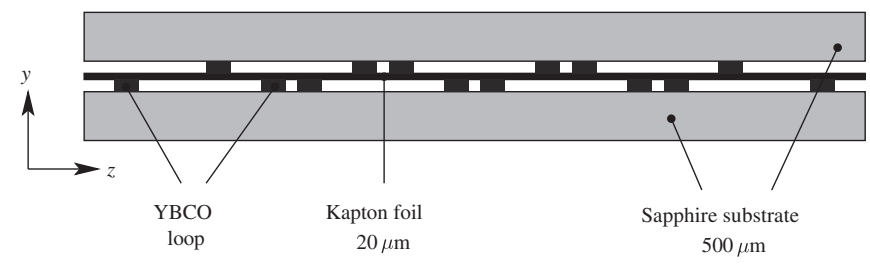

FIG. 3. Cut through the stacked induction-shimming structure with the substrate. The YBCO loops are arranged on a $500 \mu \mathrm{m}$ thick sapphire substrate. The thickness of the YBCO layer is $330 \mathrm{~nm}$. The period of the mock-up is $14 \mathrm{~mm}$.

one millimeter distance from the undulator surface. The seven loops were installed in the center of the mock-up. The arrangement of the loops is shown schematically in Fig. 3 and a photo is shown in Fig. 4. The field was measured in a helium bath cryostat-CASPER I-a field measuring device for short superconductive undulators described in detail in [9]. The result of the measurements is shown in Fig. 5. The field was measured with a Hall probe which was moved at a distance of $7.15 \mathrm{~mm} \pm$ $0.1 \mathrm{~mm}$ from the undulator surface.

As stated in [9], the calibrated Hall probe has an accuracy of $0.1 \mathrm{mT}$. The spatial resolution of the measurement equipment is $3 \mu \mathrm{m}$ and the repeatability is also about $3 \mu \mathrm{m}$. The latter was achieved by repeated measurements of a well-known undulator field, which included temperature cycling.

The position of the YBCO closed-loop system is shown at the bottom of Fig. 5. The measurement was performed

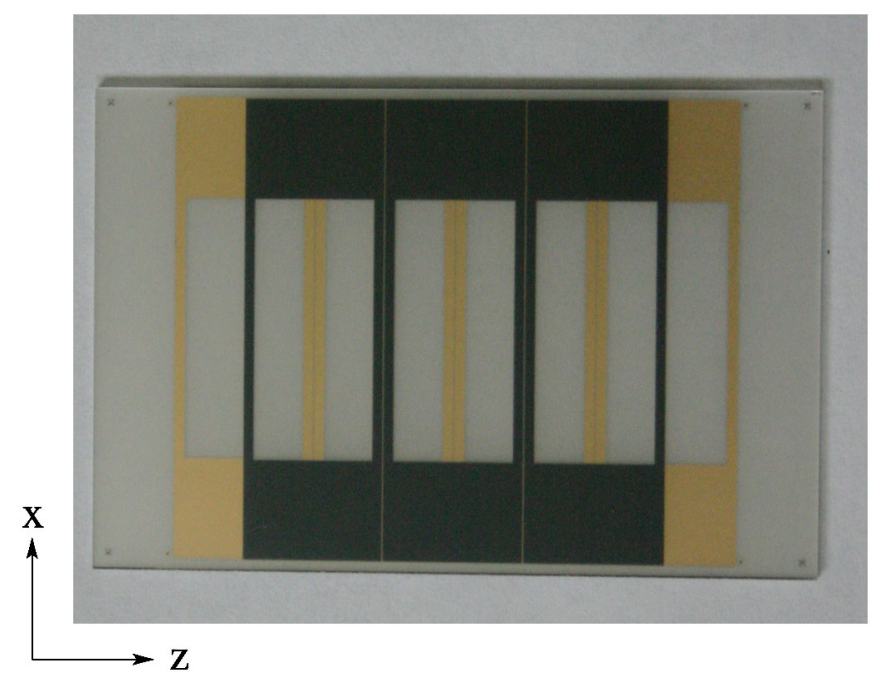

FIG. 4. (Color) Photograph of the set of seven correction loops. Two $500 \mu \mathrm{m}$ thick sapphire substrates are sputtered with four and three YBCO loops with a thickness of $330 \mathrm{~nm}$. The YBCO loops are covered with a $200 \mathrm{~nm}$ thick gold layer. The dimensions of the loops are $14 \mathrm{~mm}$ (equal to the period length of the undulator) times $44 \mathrm{~mm}$. The two substrates are stacked in the way shown in the figure and mounted at a distance of $1 \mathrm{~mm}$ from the surface of the undulator. 


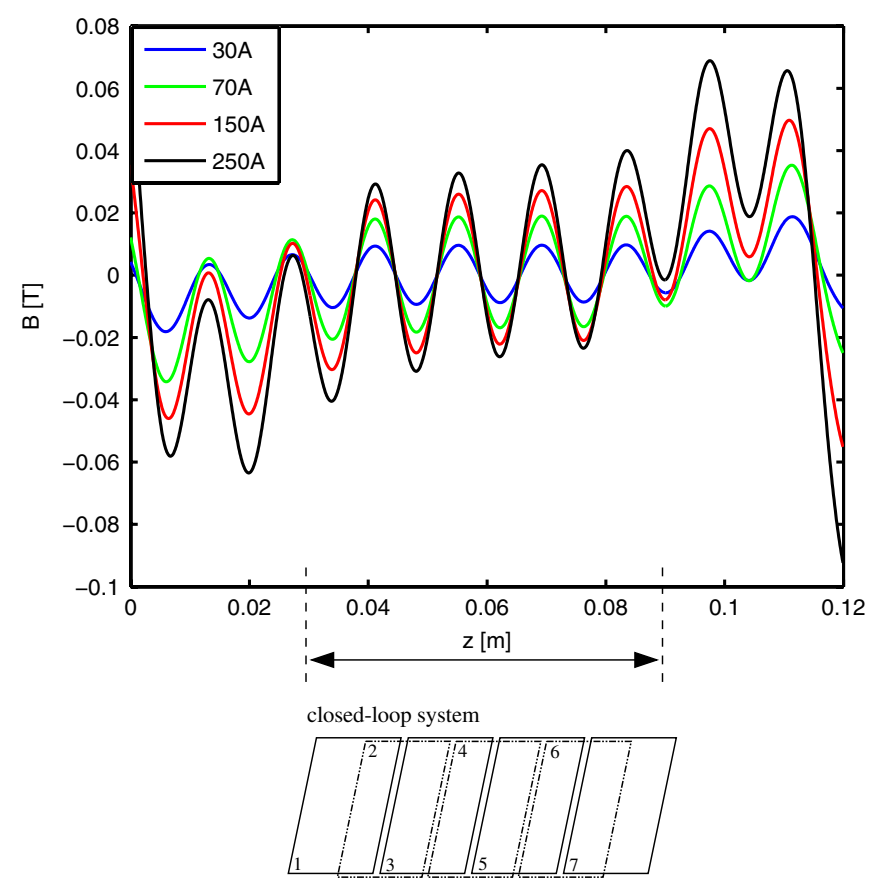

FIG. 5. (Color) The influence of the YBCO closed-loop system on the field shown in Fig. 2 for various undulator currents.

for several currents through the undulator: $I_{\text {main }}=30,70$, 100, 150, 200, and $250 \mathrm{~A}$.

Figure 5 shows that the YBCO loops flatten the field amplitudes significantly at $I_{\text {main }}=30 \mathrm{~A}$ and $I_{\text {main }}=70 \mathrm{~A}$. With increasing undulator current this effect is reduced. The difference between the fields measured with and without the YBCO loop system is shown in Fig. 6. The compensating field is positive at the left-hand side and negative at the right-hand side as one would expect from the distorted field (see Fig. 2). It also shows that both peaks change the form as the current increases. In [7] it is shown how the field compensation can be calculated with the law of Biot-Savart. This model was used to calculate the currents in the YBCO loops (Table I). The estimated accuracy of the simulated currents is about $\pm 10 \%$. Table I shows that the currents in the YBCO loops rearrange themselves

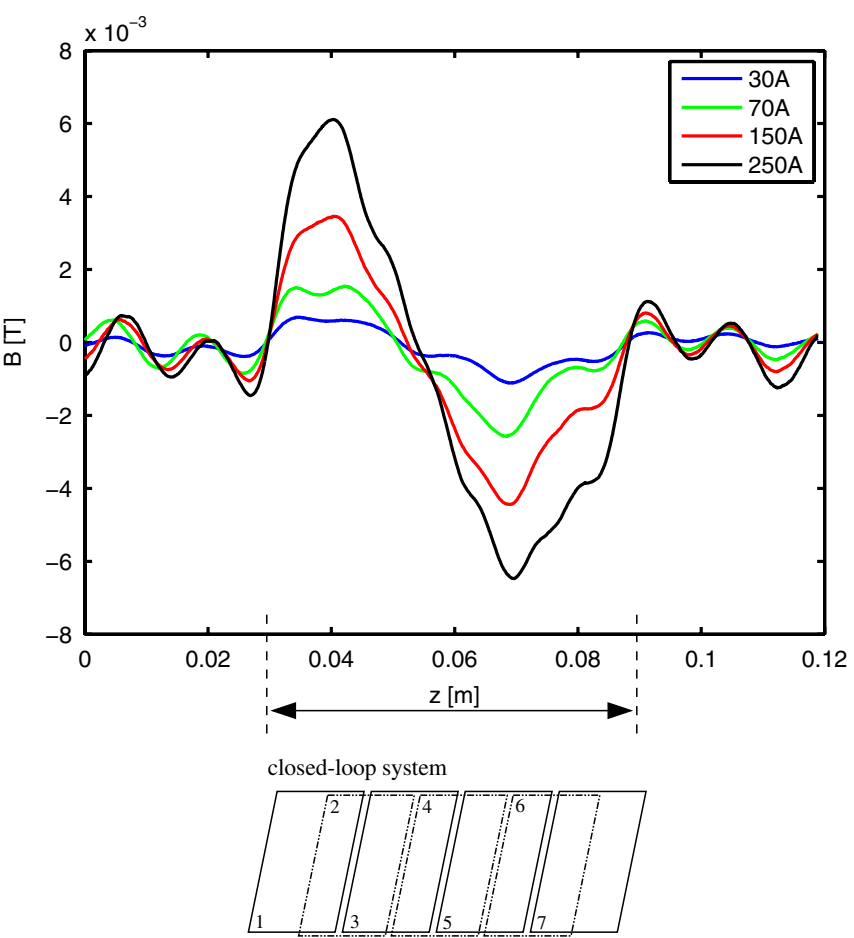

FIG. 6. (Color) Difference of the field measurements with and without the YBCO loops system. The YBCO loop system tries to compensate the field which increases from left to right in Fig. 2.

with increasing current. The maximum induced current in the loops is about $160 \mathrm{~A} \pm 10 \%$ which corresponds to a critical current density of $485 \mathrm{kA} / \mathrm{mm}^{2} \pm 10 \%$. (This value is close to the maximum critical current density of $500 \mathrm{kA} / \mathrm{mm}^{2}$ for YBCO at $4.2 \mathrm{~K}$ reported in [10].)

After reaching the level of $I_{\text {main }}=250 \mathrm{~A}$, the current is turned to $I_{\text {main }}=0 \mathrm{~A}$ in order to measure the remanent currents in the loops. The remanent currents, listed at the end of Table I, are caused by two effects: (1) eddy currents around vortices in the type-II superconductor; (2) the fact that the critical current density was reached in some loops.

In the current setup these two effects cannot be distinguished. Figure 7 shows the normalized correction fields for the different currents together with the remanent cur-

TABLE I. Simulated currents in the seven YBCO loops as a function of the current in the undulator mock-up. The error is approximately $\pm 10 \%$.

\begin{tabular}{|c|c|c|c|c|c|c|c|}
\hline \multirow{2}{*}{$\begin{array}{l}\text { Current in mock-up coil } \\
I_{\text {main }}\end{array}$} & \multicolumn{7}{|c|}{ Induced currents in closed-loop [A] } \\
\hline & 1 & 2 & 3 & 4 & 5 & 6 & 7 \\
\hline $30 \mathrm{~A}$ & 22.8 & 1.3 & 21.5 & -25.8 & 8.4 & -39.5 & -7 \\
\hline $50 \mathrm{~A}$ & 43 & -1.0 & 47 & -39 & 15.7 & -63.2 & -13 \\
\hline $70 \mathrm{~A}$ & 56 & -2.0 & 61 & -51.5 & 13.5 & -86 & -23 \\
\hline $100 \mathrm{~A}$ & 45 & 34 & 49.5 & -29 & -10 & -92.5 & -46 \\
\hline $150 \mathrm{~A}$ & 54 & 67 & 66.5 & -20.5 & -23 & -111 & -76 \\
\hline $200 \mathrm{~A}$ & 62 & 102 & 81 & -20.7 & -40 & -119.5 & -110.5 \\
\hline $250 \mathrm{~A}$ & 60 & 150 & 75 & -25.8 & -50 & -160 & -110 \\
\hline $0 \mathrm{~A}$ after $250 \mathrm{~A}$ & -3 & -4.5 & -6.7 & 6.3 & -3.5 & 14.3 & 4.5 \\
\hline
\end{tabular}




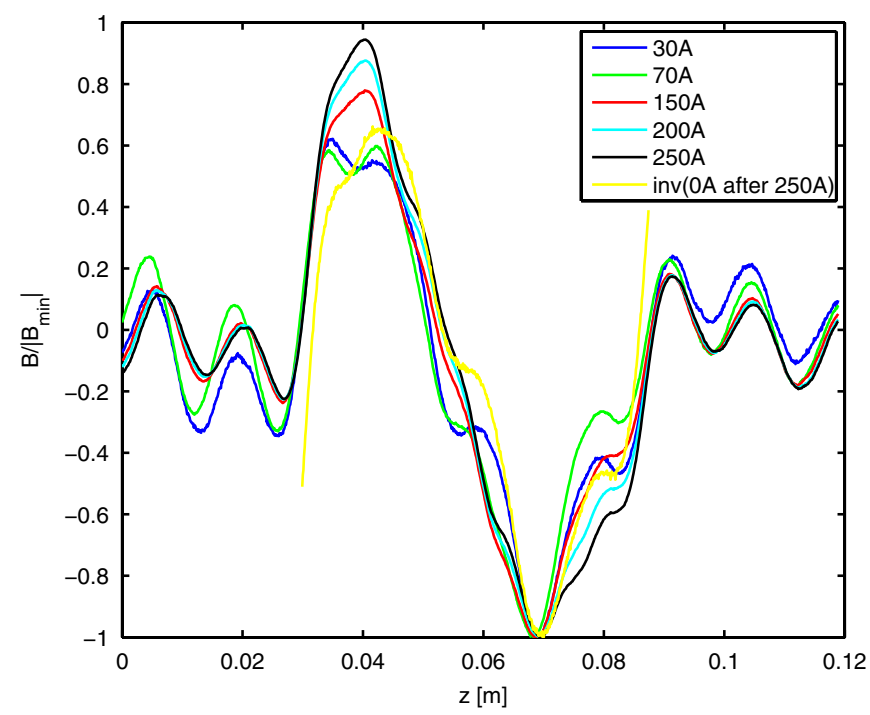

FIG. 7. (Color) Normalized field changes by the YBCO loops for the different mock-up currents shown in Fig. 6 together with the inverted remanent field after switching the mock-up current from $I_{\text {main }}=250$ to $0 \mathrm{~A}$.

rent after turning the undulator current to zero. The field direction of the remanent current was inverted. The shape of this residual field (see Fig. 7) gives a hint that the second effect is dominating here. A detailed examination of these effects is subject to a follow-up experiment.

In a shimming concept for a real undulator, the thickness of the YBCO layer has to be chosen in such a way that the induced currents stay significantly below the critical current in each part of the loop system. Then the second effect will not appear.

Another measure for the action of the loop system is the local first field integral $I_{1}(z)=\int_{Z_{\text {start }}}^{z_{\text {end }}}\left(B_{y} d z^{\prime}\right)$ along the beam trajectory in the region covered by the loops. This is shown in Fig. 8. At the beginning of the loop system the value of the integral is assumed to be 0 . The loop system reduces the first integral locally by a factor of 10 from $1.95 \times 10^{-5} \mathrm{Tm}$ to $-1.5 \times 10^{-6} \mathrm{Tm}$.

Note: the global first field integral [i.e. $\left.\int_{-\infty}^{\infty}\left(B_{y} d z^{\prime}\right)\right]$ is not changed by the induction-shimming system and has to be corrected by standard integral correctors.

Figure 9 shows the improvement in the local second field integral $\left[I_{2}(z)=\iint_{Z_{\text {start }}}^{z_{\text {end }}}\left(B_{y} d z^{\prime 2}\right)\right]$ caused by the loop system.

The efficiency of the system is shown in Fig. 10 for the different currents through the undulator. The ratio of adjacent extrema covered by the loop $j$ are compared:

$$
\Delta_{j}=|1-| \frac{A_{j, \max }}{A_{j, \min }}|| .
$$

The data are taken from the measurements shown in Fig. 5 and corresponding measurements without the inductionshimming system. The efficiency is lowest at the first loop, which suffers from a fabrication error in the YBCO layer.

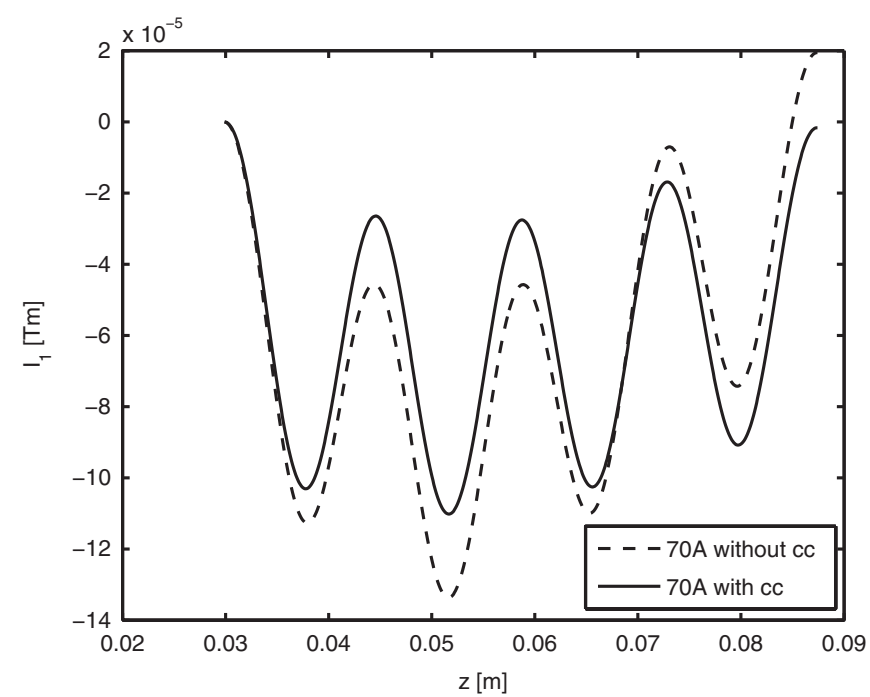

FIG. 8. Reduction of the first field integral by the loop system measured along the loop system for $I_{\text {main }}=70 \mathrm{~A}$.

The dashed curves show the ratio before compensation, the solid lines after compensation. The values of the solid lines increase towards both ends of the area covered with YBCO loops especially for currents above $I_{\text {main }}=150 \mathrm{~A}$. This is caused by two effects: (1) The induced currents reach the saturation current, in the right loops for $150 \mathrm{~A} \leq I_{\text {main }} \leq$ $250 \mathrm{~A}$ (see Table I) and in the first loop at the left-hand side at $I_{\text {main }} \gtrsim 70$ A due to a fabrication error. This is the cause for the higher values at point 1 at the left-hand side of Fig. 10. (2) At both ends of the loop system the values in Fig. 10 are higher since the outmost loops have no partner due to the finite length of the induction-shimming system (see Figs. 3 and 4).

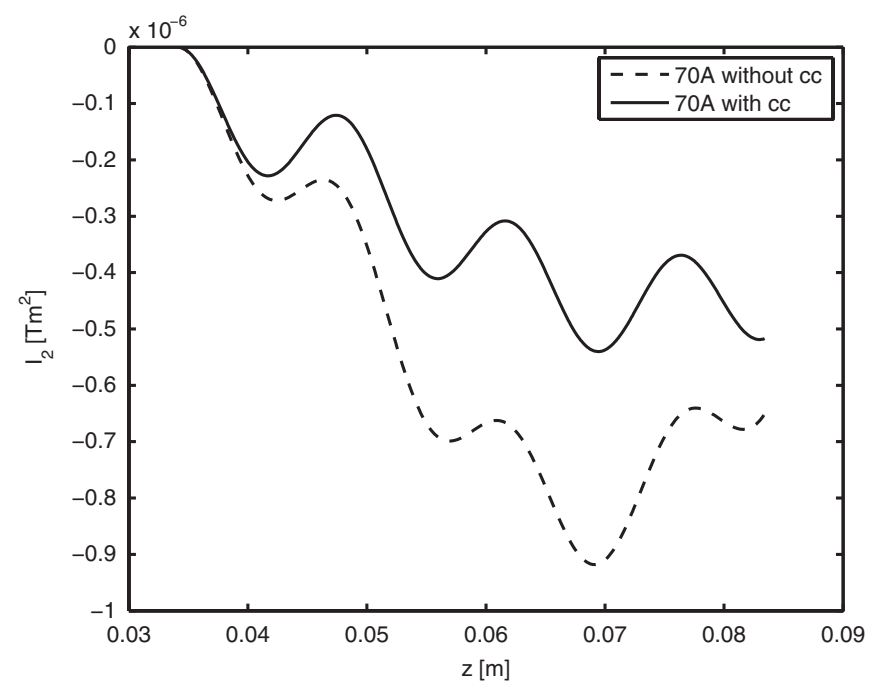

FIG. 9. Improvement in the second field integral caused by the loop system for $I_{\text {main }}=70 \mathrm{~A}$. The second field integral was calculated from the first to the last extremum covered by the loop system. 


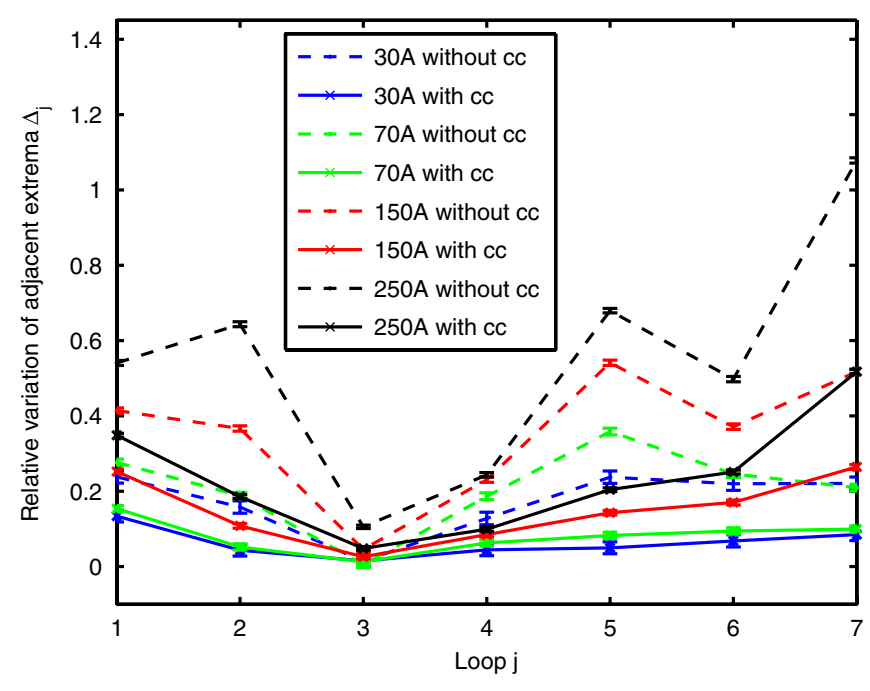

FIG. 10. (Color) Ratio of the absolute values of adjacent extrema with and without the YBCO loop system for the different currents $I_{\text {main }}$ of Table I. $\Delta_{j}=|1-| \frac{A_{j, \max }}{A_{j, \min }}||$ with the loop number $j$. The values at the beginning and the end are higher due to the finite length of the induction-shimming system.

Another measure for the increase of the field quality caused by the induction-shimming system is the relative variation of the field gradients at adjacent turning points of the undulator field,

$$
\Delta_{i}=|1-| \frac{G_{i}}{G_{i+1}}||
$$

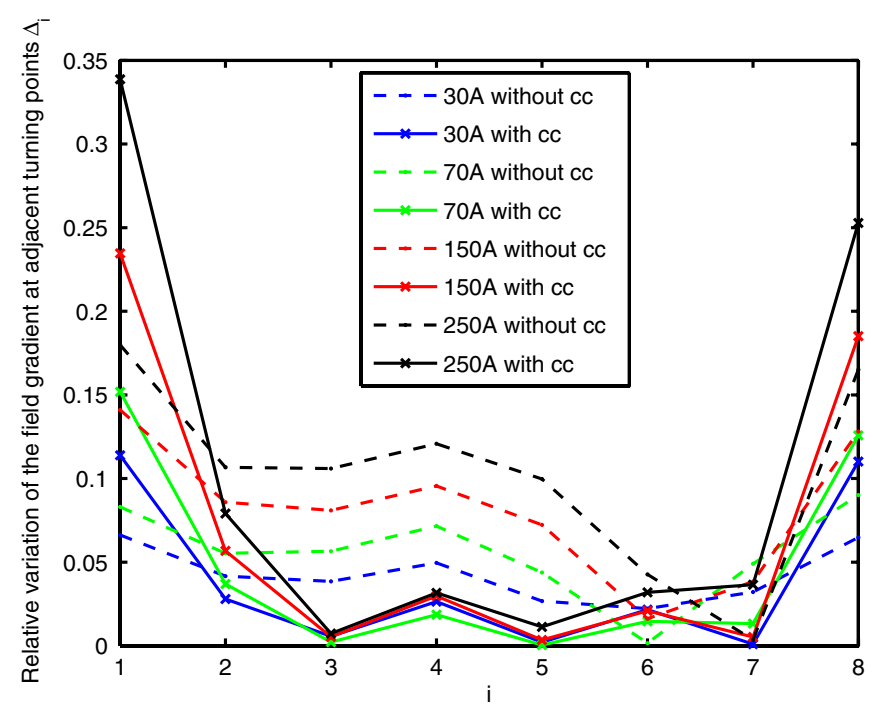

FIG. 11. (Color) Ratio of the absolute field gradients at adjacent turning points with and without the YBCO loop system for the different currents $I_{\text {main }}$ of Table I. $\Delta_{i}=|1-| \frac{G_{i}}{G_{i+1}}||$ with the turning point pair $i$. The significant increase at the beginning and end is due to the finite length of the induction-shimming system. The first (last) turning point is at the beginning (end) of the first (last) loop of the induction-shimming system. with the turning point pair $i$, as shown in Fig. 11. The first and last turning points of the magnetic field used in this comparison are located at the beginning of the first and end of the last loop.

Without the loop system (dashed lines), the variation of the field gradient increases proportionally to the mock-up current $I_{\text {main }}$. The induction-shimming system reduces this variation to a significant lower level $\left(\Delta_{i} \lesssim 4 \%\right)$ for all mock-up currents and $3 \leq i \leq 7$ (solid lines). At the beginning and the end of the loop system, this effect is reversed due to the finite length of the loop system.

\section{CONCLUSION}

This demonstration experiment shows that the phase error in superconductive undulators can be significantly reduced by an array of coupled HTSC closed loops. The measured correction field of the loop system was about $6 \mathrm{mT}$ at a distance of $7.15 \pm 0.1 \mathrm{~mm}$. Typically an undulator with a period length of $14 \mathrm{~mm}$ has a gap of $5 \mathrm{~mm}$, resulting in a maximum on axis field of $1.5 \mathrm{~T}$. In that case the loop system will only be $2.5 \mathrm{~mm}$ away from the beam trajectory. The correction field scales with $1 / r$ and therefore the available correction field will be about $17 \mathrm{mT}$. In a real superconductive undulator the field error should not exceed $1 \%$, i.e., $15 \mathrm{mT}$ for the example mentioned above. With correction loops on both undulator coils, the available correction field is $34 \mathrm{mT}$ and sufficient for a good correction. The YBCO loops with a thickness of $330 \mathrm{~nm}$ are sufficient for most superconductive undulators. In this experiment both the four and the three YBCO loops are sputtered on a $500 \mu \mathrm{m}$ thick substrate as shown in Fig. 4. In a practical application of this principle, the thickness of the substrate has to be reduced. This can be done by using only one substrate with loops on both sides of the substrate and a thinner substrate similar to the one used for HTSC cables.

\section{OUTLOOK}

A follow-up experiment to quantify the effect of induction shimming on the phase error is currently in preparation. A new 12.5 full period mock-up undulator with $8 \mathrm{~mm}$ magnetic gap and reasonable field quality will be equipped with two induction-shimming systems (one per coil). Each system consists of 24 YBCO closed loops deposited on sapphire wafers. The magnetic field will be mapped with Hall probes mounted on a sled moving in the gap between the two mock-up coils.

\section{ACKNOWLEDGMENTS}

The authors want to thank D. Erbe, who designed and constructed important parts of the measurement setup. The authors also want to thank R. Semerad and his colleagues from the company THEVA Dünnschichttechnik $\mathrm{GmbH}$, Ismaning, Germany, for the fabrication of the induction- 
shimming test device.

[1] Undulators, Wigglers and their Applications, edited by $\mathrm{H}$. Onuki and P. Elleaume (Taylor \& Francis, London, 2003), Chap. 5, Vol. 1, pp. 148-213, ISBN 0-415-28040-0.

[2] J. Clarke, The Science and Technology of Undulators and Wigglers (Oxford Science Publication, New York, 2004).

[3] S. Chouhan, R. Rossmanith, S. Strohmer, D. Doelling, A. Geisler, A. Hobl, and S. Kubsky, in Proceedings of the 20th Particle Accelerator Conference, Portland, OR, 2003 (IEEE, New York, 2003), pp. 899-901.

[4] S. Prestemon, D. Dietderich, S. Bartlett, M. Coleman, S. Gourlay, A. Lietzke, S. Marks, S. Mattafirri, R. Scanlan, R. Schlueter, B. Wahrer, and B. Wang, IEEE Trans. Appl. Supercond. 15, 1236 (2005).

[5] D. Wollmann, A. Bernhard, S. Casalbuoni, M. Hagelstein, B. Kostka, R. Rossmanith, M. Weisser, E. Steffens, G.
Gerlach, and T. Baumbach, in Proceedings of the 10th European Particle Accelerator Conference, Edinburgh, Scotland, 2006 (EPS-AG, Edinburgh, Scotland, 2006), pp. 3577-3579.

[6] D. Doelling, A. Hobl, H.-U. Klein, D. Krischel, P. Komorowski, and M. Meyer-Reumers, in Proceedings of the 2007 Particle Accelerator Conference, Albuquerque, New Mexico, 2007 (IEEE, Albuquerque, New Mexico, 2007), pp. 935-937.

[7] D. Wollmann, A. Bernhard, P. Peiffer, T. Baumbach, and R. Rossmanith, Phys. Rev. ST Accel. Beams 11, 100702 (2008).

[8] D. Wollmann, A. Bernhard, P. Peiffer, R. Rossmanith, and T. Baumbach, in Proceedings of the 11th European Particle Accelerator Conference, Genoa, 2008 (EPS-AG, Genoa, Italy, 2008), pp. 2323-2325.

[9] E. Mashkina et al., J. Phys. Conf. Ser. 97, 012020 (2008).

[10] B. Roas, L. Schultz, and G. Saemann-Ischenko, Phys. Rev. Lett. 64, 479 (1990). 